${ }^{1}$ Universitätsklinik für Hals-, Nasen- und Ohrenheilkunde und Poliklinik, Universitätsklinikum Freiburg

${ }^{2}$ Sektion Neuroradiologie, Neurozentrum, Universitätsklinikum Freiburg

\title{
Computerassistierte Chirurgie (CAS) zur optimalen Behandlung der kranio- fazialen fibrösen Dysplasie
}

D ie fibröse Knochendysplasie stellt eine relativ häufige Fehlentwicklung des knochenbildenden Mesenchyms dar, wobei das Knochenmark durch fibröses Mark ersetzt wird und Faserknochen bei ausbleibender Transformation in lamellären Knochen bestehen bleibt [1]. Die Knochenherde können monoostotisch oder polyostotisch auftreten. Bei der monoostotischen Form ist u. a. vorwiegend der Schädel und der Kiefer befallen, wobei nach Adler der Schädel in $14 \%$ und der Kiefer in $11 \%$ be- troffen ist [1]. Bei der kraniofazialen Lokalisation können die Knochenauftreibungen zu kosmetisch-ästhetischen sichtbaren Deformierungen und lokalen Schwellungen, zu Verlegungen der Drainagewege des Nasennebenhöhlensystems und neurologischen Kompressionssyndromen führen [16].

Neben dem psychischen Leidensdruck aufgrund der ungewollten Aufmerksamkeit und Neugierde des sozialen Umfeldes bei äußerlich sichtbaren unnatürli- chen Deformierungen des Gesichts- und Hirnschädels resultieren klinische Symptome wie lokalisierter Knochenschmerz bis hin zu diffusem Gesichtsschmerz und umschriebenen Missempfindungen. Letztere sind teilweise bedingt durch Kompression der Hirnnerven an deren Foramina

Auszugsweise vorgetragen anläßlich der 10. Jahrestagung der Deutschen Gesellschaft für Schädelbasischirurgie, 8.-9. November 2002, Heidelberg

\begin{tabular}{|c|c|c|c|c|c|}
\hline \multicolumn{6}{|c|}{ Patientendaten } \\
\hline Fall & $\begin{array}{l}\text { Patient, Alter, } \\
\text { Geschlecht }\end{array}$ & $\begin{array}{l}\text { Lokalisation der kraniofazialen } \\
\text { fibrösen Dysplasie }\end{array}$ & Klinische Symptome & Therapie & CAS \\
\hline 1 & A.A., 47, w. & $\begin{array}{l}\text { Aufsteigender Unterkieferast, Maxilla, } \\
\text { Os zygomaticum rechts, Zustand nach } \\
\text { Vor-Op. alio loco, Fazialisparese }\end{array}$ & $\begin{array}{l}\text { Kosmetisch unbefriegend, } \\
\text { diffuser Gesichtsschmerz }\end{array}$ & Abtragung von Unterkieferknochen & Ja \\
\hline 2 & D.S., 35, w. & Stirnhöhle rechts & $\begin{array}{l}\text { Lokaler Kopfschmerz, } \\
\text { Missempfindungen }\end{array}$ & $\begin{array}{l}\text { Radikale Stirnhöhlen-Op. nach } \\
\text { Riedel, sekundäre Rekonstruktion } \\
\text { durch ein Titan-CAD-CAM-Implantat }\end{array}$ & Ja \\
\hline 3 & S.M., 35, m. & Felsenbein rechts & Lokaler Kopfschmerz,Vertigo & $\begin{array}{l}\text { Mastoidektomie, } \\
\text { Saccusdekompression }\end{array}$ & Nein \\
\hline 4 & W.E., 32, $\mathrm{m}$. & Naseneingang, Sinus maxillaris rechts & $\begin{array}{l}\text { Lokaler Gesichtsschmerz, } \\
\text { Nasenatmungsbehinderung, } \\
\text { Kompression des N. } V_{2} \text { rechts }\end{array}$ & $\begin{array}{l}\text { Naseneingangserweiterung, } \\
\text { Caldwell-Luc-Operation rechts }\end{array}$ & Nein \\
\hline 5 & G.E., 23, w. & Keilbeinhöhle rechts & Lokaler Kopfschmerz, Mukozele & $\begin{array}{l}\text { Transnasale Keilbeinhöhlen-Op. } \\
\text { rechts }\end{array}$ & Nein \\
\hline 6 & G.C., 26, w. & Siebbein rechts & $\begin{array}{l}\text { Rezidivierender lokaler } \\
\text { Kopfschmerz }\end{array}$ & $\begin{array}{l}\text { Keine Op., Beobachten: 1- bis } \\
\text { 2-jährliche MR-Untersuchungen }\end{array}$ & - \\
\hline
\end{tabular}



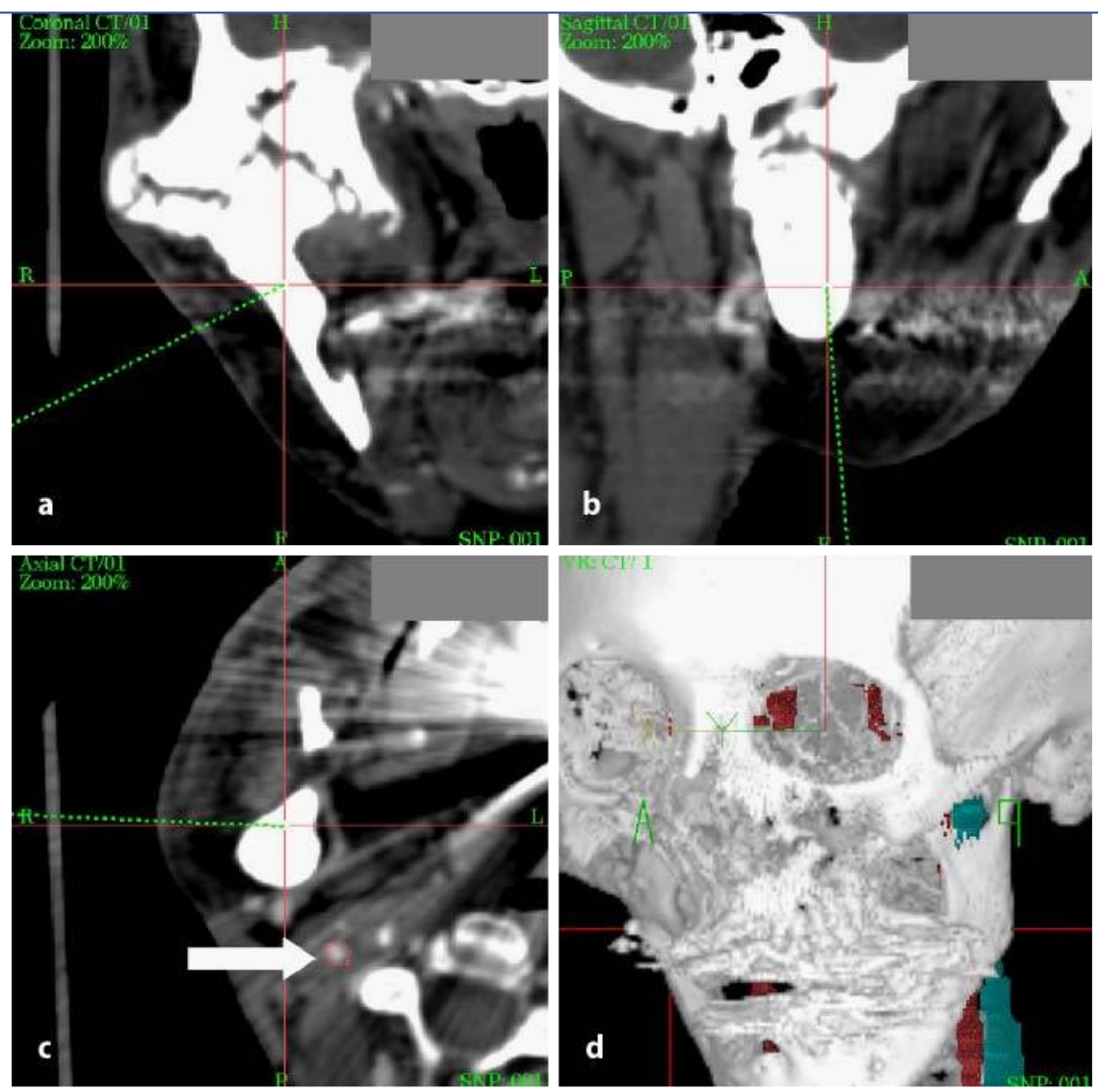

Abb. 1a-d $\triangle$ 3D-Rekonstruktion (d) mit korrespondierender koronarer Schicht (a), sagittaler Schicht (b) und axialer Schicht (c) eines 3D-CT-Datensatzes zur intraoperativen Navigation (System Stryker-Leibinger). Dargestellt ist der durch die fibröse Dysplasie fibrotisch aufgetriebene aufsteigende Unterkieferast (Patientin 1, - Tabelle 1), der zur Gegenseite symmetrisch navigiert kontrolliert mit der Knochenfräse reduziert wird. Das Fadenkreuz zeigt die Pointerspitze, die unterbrochene Linie die gehaltene Richtung des Pointers. Der Pfeil in c zeigt auf die rot konturierte A. carotis. Die A. carotis ist in d rot, die V. jugularis blau konturiert

oder durch sekundäre Muko- oder Pyozelen im Nasennebenhöhlenbereich infolge einer Verlegung der Drainagewege [17]. Ebenso können Sehstörungen bis zur Erblindung, Augenmotilitätsstörungen mit Doppelbildern oder eine Protrusio bulbi beobachtet werden.

Eine kausale Therapie der fibrösen Dysplasie ist bislang nicht bekannt [17]. Die Behandlung der fibrösen Dysplasie erfolgt daher nur konservativ beobachtend oder nach sorgfältiger Indikationsprüfung chirurgisch-symptomatisch bei Nachweis klinischer Symptome. Eine radikale chirurgische Resektion des befallenen Knochens ist bei der kraniofazialen fibrösen Dysplasie nur sehr selten möglich aufgrund der unmittelbaren topographischen Nachbarschaft zu vitalen Nerven- und Gefäßstrukturen [25].

Ziel einer chirurgisch-symptomatischen Therapie ist die Wiederherstellung der ursprünglichen annähernd symmetrischen äußerlich sichtbaren kraniofazialen Außenkonturen, die Wiedereröffnung der nasalen und paranasalen Drainagewege und die Dekompression neurovaskulärer Strukturen durch kontrolliertes Abtragen des überschüssig gewachsenen degenerativen Knochens. Bei der knöchernen Wiedereröffnung der paranasalen Drainagewege oder der knöchernen Dekompression neurovaskulärer Strukturen ist der Endpunkt der operativen Manipulation eindeutig definiert durch die Wiederherstellung der ursprünglichen physiologischen Funktion.

Anders ist die Situation bei der Korrektur durch überschüssiges Knochenwachstum entstandener Knochendeformitäten im sichtbaren Bereich des Gesichts- und Hirnschädels, da hierbei keine physiologischen Körperfunktionen, sondern die ursprüngliche, annähernd symmetrische
Gesichts- und Hautkontur wiederhergestellt werden soll. Intraoperativ fehlen dafür die Bemessungsgrundlagen- und grenzen als anatomische Landmarken, um den Endpunkt der notwendigen chirurgischen Manipulationen in Form kontrollierter Knochenabtragung definieren zu können. Sowohl zu wenig als auch zu viel abgetragener Knochen würde nur eine weitere sichtbare Deformierung des sichtbaren Gesichts- und Hirnschädels bedeuten.

In diesen Fällen kann der Einsatz der computerassistierten Chirurgie (CAS) hilfreich sein. Die CAS dient dabei nicht nur zur intraoperativen Kontrolle der Knochenabtragungsgrenzen, sondern auch zur Planung möglicher Implantate als Defektdeckung nach knöchernen radikal-chirurgischen Maßnahmen. In einer retrospektiven Qualitätssicherungsanalyse stellen wir die Indikation zur CAS bei Patienten mit kraniofazialer Dysplasie dar.

\section{Patienten und Methoden}

Patienten. Vom 1.1.1999 bis zum 1.1.2004 stellten sich 6 Patienten mit einer kraniofazialen monoostotischen fibrösen Dysplasie bei uns vor. Von diesen 6 Patienten im Alter zwischen 23 und 47 Jahren waren 4 weiblich und 2 männlich (• Tabelle 1 ). Klinisches Leitsymptom war ein lokalisierter oder diffuser Kopf- oder Gesichtsschmerz. Bei einem Patienten mit einer kraniotemporalen Manifestation einer fibrösen Dysplasie fiel neben dem Kopfschmerz zusätzlich auch eine im Intervall auftretende ungerichtete Vertigo auf. Computertomographisch war eine für die fibröse Dysplasie charakteristische Sklerosierung im Bereich des Saccus endolymphaticus auffällig. Die Diagnose erfolgte anhand eines hochauflösenden Schädel-CT.

Radiologische Kriterien für eine fibröse Dysplasie waren das Milchglasphänomen als klassisches Kennzeichen der fibrösen Dysplasie, wobei dieses Phänomen von der Beschaffenheit der Matrix und deren Mineralisation abhängt, sodass diese Läsionen auch zystisch oder unterschiedlich stark ausgeprägte Sklerosierungen aufweisen können. Zum Ausschluss einer polyostotischen Variante der fibrösen Dysplasie wurden Röntgenzielaufnahmen der Extremitäten mit Fokus auf die 


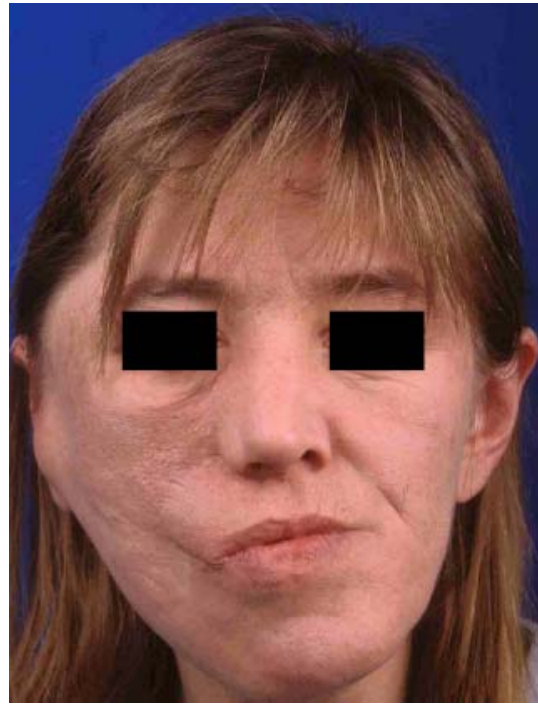

Abb. 2 × Patientin präoperativ mit einer alio loco bereits mehrfach voroperierten monoostotischen fibrösen Dysplasie der Maxilla, des aufsteigenden Unterkieferastes, des Os zygomaticum und einer iatrogenen rechtsseitigen Fazialisparese (Patientin 1, $0 \mathrm{Ta}-$ belle 1)

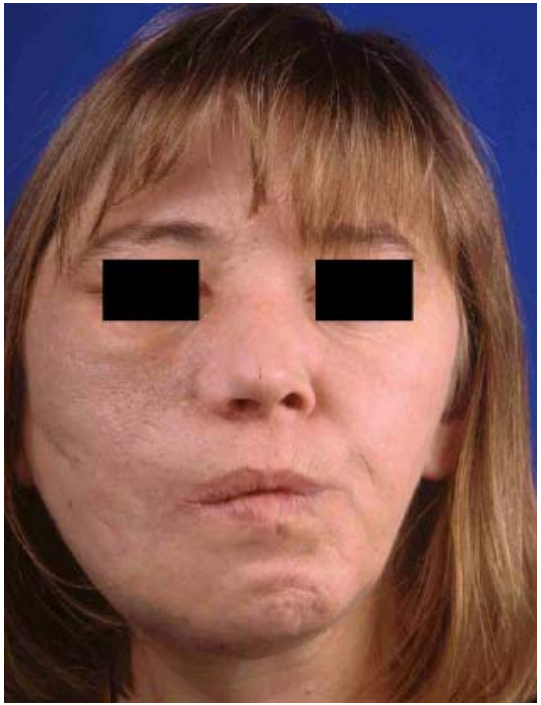

Abb. $3 \Delta$ Patientin eine Woche postoperativ nach zur Gegenseite symmetrisch navigiert kontrollierter Knochenabtragung mit einer alio loco bereits mehrfach voroperierten monoostotischen fibrösen Dysplasie der Maxilla, des aufsteigenden Unterkieferastes, des Os zygomaticum und einer iatrogenen rechtsseitigen Fazialisparese (Patientin 1, - Tabelle 1). Die seit Jahren bestehende Fazialisparese wurde durch eine statische Fascia-lata-Plastik aufgehängt und am knöchern modellierten Os zyogomaticum korrigiert. Die operationsbedingte Weichteilschwellung der rechte Gesichtshälfte ist noch nicht ganz abgeklungen

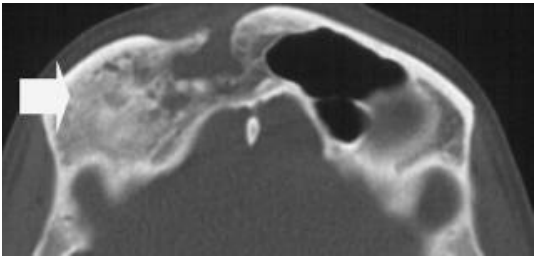

Abb. $4 \triangle$ Axiale CT-Schicht im Knochenfenster einer monoostotischen fibrösen Dysplasie des rechsseitigen Os frontale mit Abschottung eines residualen rechtsseitigen Sinus frontalis und äußerlich sichtbaren Deformität der Glabella (Patientin 2, - Tabelle 1). Morphologisch liegt kein typisches Milchglasphänomen vor, vielmehr sind zystische Veränderungen mit diskreten Sklerosierungen dominierend. Im Bereich der Glabella ist, eher untypisch, die Kontinuität des Periost aufgehoben

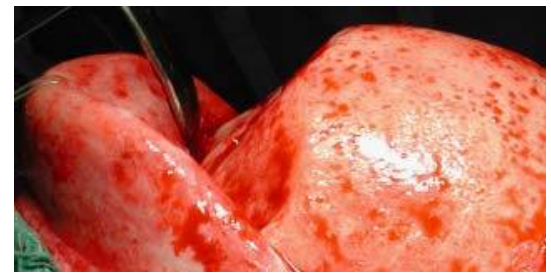

Abb. $\Delta \Delta$ Intraoperativer Situs nach Resektion der fibrotisch deformierten Stirnhöhle (Stirnhöhlenoperation nach Riedel) (Patientin 2, - Tabelle 1). Mit Hilfe eines postoperativ angefertigten CAS-CT-Datensatzes wird ein CAD-CAM-Implantat aus Titan passgenau fabriziert (Fa. Cranio Construct GmbH, Bochum)

entenkopfs haben wir mit Hilfe der Oberfächenregistrierung erfasst. Analog zur digitalen Operationsplanung wurden bei einem anderen Patienten nach kompletter radikal-chirurgischer Sanierung des befallenen Os frontale (Radikal-Op. nach Riedel) zur Defektrekonstruktion zweizeitig das zu fabrizierende Implantat konfiguriert. Der so konfigurierte Datensatz wurde anschließend zur Herstellung des Implantates an die Fa. Cranio Construct $\mathrm{GmbH}$, Bochum, geschickt. Dort wurde nach den entsprechenden Vorgaben ein passgenaues CAD-CAM-Titan-Implantat angefertigt und an uns zurückgesendet.

\section{Ergebnisse}

Die Möglichkeit zu einer ausschließlich symptomatischen chirurgischen Therapie bei der kraniofazialen fibrösen Dysplasie verlangt eine sehr sorgfältige Abwägung zwischen der operativen Morbidität und 
dem langfristig für den Patienten zu erwartenden Vorteil einer solchen Maßnahme. Nur wenn unverkennbare gravierende kosmetisch-ästhetisch Veränderungen des sichtbaren Gesichts- und Hirnschädels vorlagen bzw. ein den Patienten in seinen täglichen Lebensfunktionen nachweisbar einschränkender, nicht mehr tolerabler Kopf- oder Gesichtschmerz oder ein neurologisch verifizierbares Kompressionssyndrom im Hirnnervenbereich vorlag, haben wir die Indikation zu einer operativen Intervention gestellt.

Ziel der Operation war zum einen eine knöcherne Dekompression betroffener Hirnnerven an deren knöchernen Austrittspunkten am Splanchno- oder Neurokranium und die Freilegung und Wiederherstellung der ursprünglichen nasalen und paranasalen Drainagewege (Patienten 4, 5, - Tabelle 1). Die Operationen erfolgten zur Vermeidung von Narben am Gesichtsschädel ausschließlich transfacial über ein modifiziertes hemilaterales Midfacial Degloving (Patient 4, - Tabelle 1) oder transnasal (Patientin 5, - Tabelle 1).

Im Falle einer temporofazialen fibrösen Dysplasie haben wir zur Dekompression eines fibrotisch ossär veränderten Saccus endolymphaticus einen konventionellen transmastoidalen operativen Zugang gewählt. Bei einer anderen Patienten stand die kosmetisch-ästhetische Rehabilitation bei einer rechtsseitigen hemilateralen monoostotischen fibrösen Dysplasie der Mandibula, Maxilla und des Os zygomaticum im Vordergrund. Die Patienten hatte bereits zahlreiche Voroperationen alio loco hinter sich (Patientin 1, • Tabelle 1).

Bei einer der vorangegangenen Operation kam es zu einer bleibenden kompletten ipsilateralen Facialisparese (Grad VI nach House-Brackman; - Abb. 2). Mit Hilfe der CAS konnte der überschüssig befallene Knochen kontrolliert in Symmetrie zur korrespondierenden Gegenseite mit der Bohrfräse abgetragen werden (- Abb. 1a-d). Durch die Operation möglicherweise gefährdete vitale Nerven- und Gefäßstrukturen wie die in unmittelbarer Nachbarschaft liegende A. carotis wurden in der CAS visualisiert.

Durch die zahlreichen Voroperationen war das Operationsgebiet bereits sehr vernarbt und typische knöcherne Landmarken durch das überschüssige Knochen-

\section{J. Schipper · W. Maier · A. Berlis · G. J. Ridder}

\section{Computerassistierte Chirurgie (CAS) zur optimalen Behandlung der kraniofazialen fibrösen Dysplasie}

\section{Zusammenfassung}

Hintergrund. Behandlungsbedürftigkeit bei der seltenen Knochenerkrankung der kraniofazialen fibrösen Dysplasie besteht lediglich bei sichtbaren Deformitäten des Gesichts- und Hirnschädels, Kompressionssyndromen und Verlegung der nasalen und paranasalen Drainagewege.

Methodik und Patienten. In einer retrospektiven Untersuchung von 6 Patienten mit kraniofazialer monoostotischer fibröser Dysplasie wurde analysiert, bei welchen operativen Indikationen die computerassistierte Chirurgie (CAS) hilfreich sein kann.

Ergebnisse und Fazit. Die CAS dient als intraoperative Bemessungsgrundlage zur Korrektur sichtbarer Knochendeformierungen des Hirn- und Gesichtsschädels und erlaubt in Symmetrie zur Gegenseite ein kon-

\section{Computer-assisted surgery for a calculated bone resection in craniofacial fibrous dysplasia}

\section{Abstract}

Background and objective. The rare bone disease craniofacial fibrous dysplasia is only treated in cases of visible deformities of the splanchnocranium and neurocranium, compression syndromes, and delocation of nasal and paranasal drainage.

Methods and patients. In a retrospective quality assessment analysis of six patients with craniofacial monostotic fibrous dysplasia, the indications were analysed for situations in which computer-assisted surgery (CAS) might be helpful.

Results and conclusions. CAS serves as an intraoperative basis for the assessment of cosmetic-aesthetic corrections of visible bone deformities of the splanchnocranium and neurocranium, and allows the controlled removal of pathologically affect- trolliertes Abtragen des pathologisch veränderten Knochens. Besonders an der Frontobasis ist dies von Bedeutung, da eine neu auftretende Gesichtsdeformierung zu einer hohen psychosozialen Belastung führen kann. Ferner ermöglicht die CAS das Konfigurieren von Implantaten zur Defektrekonstruktion nach knöchernen radikalchirurgischen Herdsanierungen. Nach den Datensätzen lassen sich passgenaue Implantate zur Defektdeckung präfabrizieren.

\section{Schlüsselwörter}

Fibröse Dysplasie · Nasennebenhöhlenerkrankungen - Kompressionsyndrom . Defektrekonstruktion .

Computerassistierte Chirurgie

ed bone in contralateral symmetry. This seems to be important, especially at the anterior skull base, as deformities of the human face produce strong psychological suffering. Furthermore, CAS allows the configuration of implants for defect reconstruction after focal restorations with bony radical surgery. Implants of various material can be prefabricated from these data, which can then be exactly fitted to cover the defect.

\section{Keywords}

Fibrous dysplasia - Paranasal sinus diseases - Nerve compression syndromes . Reconstructive surgical procedures . Computer-assisted surgery 
wachstum im Rahmen der fibrösen Dysplasie nicht mehr nutzbar, sodass eine konventionelle anatomisch-chirurgische Präparation unmöglich war. Die intraoperative Orientierung erfolgte daher ausschließlich durch die CAS. Zusätzlich wurde auf Wunsch der Patienten die Fazialisparese durch ein statisches Fascia-lata-Transplantat korrigiert (• Abb. 3). Eine präoperative elektroneuronographische Untersuchung der mimischen Muskulatur zeigte bereits eine Denaturierung der motorischen Endplatten, sodass auch eine mögliche M.-gracilis-Plastik ausgeschlossen war.

Der operative Zugangsweg erfolgte präaurikulär, transparotideal. Bei einer weiteren Patientin (Patientin 2, - Tabelle 1) haben wir eine komplette radikal-chirurgische Sanierung des deformierten und schmerzenden Ossa frontalia bei fibröser Dysplasie durchgeführt (• Abb. 4, 5). Zur zweizeitigen Defektdeckung haben wir mit Hilfe der CAS ein zu fabrizierendes Implantat konfiguriert. Nach unseren Angaben wurde dazu ein CAD-CAM-Titan-Implantat hergestellt, das sich detailgetreu einpassen ließ (• Abb. 6). Der Zugang erfolgte über einen bikoronaren $\mathrm{Zu}$ gang nach Unterberger, sodass im sichtbaren Gesichtsbereich keine Narben erzeugt wurden (• Abb. 7).

Alle operierten Patienten waren postoperativ schmerz- und beschwerdefrei. Die histologische Analyse der Operationspräparate bestätigte in allen Fällen die präoperative Verdachtsdiagnose. Eine andere Patientin (Patientin 6, - Tabelle 1) mit einer im rechten Siebbeinbereich lokal begrenzten fibrösen Dysplasie rieten wir nach einem ausführlichen Aufklärungsgespräch über die Gutartigkeit dieser Erkrankung von einer operativen Intervention zunächst ab, da die beklagten Beschwerden bislang von der Patienten selbst als tolerabel empfunden wurden. Neurologisch fanden sich keinerlei Hinweise auf ein mögliches Kompressionssyndrom. Wir rieten ihr in Abständen von 1-2 Jahren oder bei akuter Beschwerdezunahme zur MR-Kontrolle.

\section{Diskussion}

Friedrich Daniel von Recklinghausen (1833-1910) beschrieb 1891 untypische Ver- änderungen von Knochenstrukturen, die schließlich 1938 durch Louis Lichtenstein (1906-1977) als fibröse Dysplasie charakterisiert wurden [15]. Ergänzt wurde diese Publikation einige Jahre später durch eine gemeinsame Arbeit der amerikanischen Pathologen Henry Lewis Jaffé (18961979) und Louis Lichtenstein im Jahr 1942 $[12,13]$. Die fibröse Dysplasie wird daher auch synonym als Morbus Jaffé-Lichtenstein bezeichnet. Unterschieden wird eine monoostotische Variante [9] mit einer lokal begrenzten knöchernen Manifestation und eine polyostotische Form der fibrösen Dysplasie mit einer multilokulären Osteosklerose [3].

Das McCune-Albright-Syndrom wurde bereits 1937 erstmals beschrieben [2] als eine sklerosierende Knochenerkrankung kombiniert mit einer Endokrino-pathie. Café-au-lait-Flecken der Haut, $\mathrm{Pu}$ bertas praecox, eine Akromegalie und fakultativ ein Hyperthyroidismus bzw. eine Hashimoto-Thyreoiditis sind charakteristisch. 1992 konnte eine Punktmutation auf Chromosom 20 (20q12-q13) für das $\mathrm{McCu}$ ne-Albright-Syndrom nachgewiesen werden $[16,23,30]$. Dies deutet daraufhin, das es sich auch bei der fibrösen Dysplasie womöglich um eine genetisch determinierte hormonelle Stoffwechselerkrankung handelt. Unterstützt wird diese Annahme dadurch, dass die fibröse Dysplasie in der 1. und 2. Lebensdekade auftritt - mit einer Prävalenz für das weibliche Geschlecht - und nach Abschluss der Pubertät in $60-80 \%$ der Fälle sistiert [7].

Behandlungsversuche mit einer additiven Therapie mit Kalzium, Calcitonin und Vitamin D waren jedoch bislang ohne nachweisbaren Erfolg [29]. Eine fehlende kausale Therapie oder die Möglichkeit zu einer radikalchirurgischen Sanierung des befallenen Knochens bedingt eine entsprechende hohe Rezidivrate. Maßnahmen einer knöchernen chirurgischen Herdsanierung sollten daher nur bei offensichtlichen Nachweis klinischer Symptome wie Nervenkompression, pathologischen Spontanfrakturen, Verlegung nasaler oder paranasaler Drainagewege sowie sichtbarer Entstellungen des Splanchnound Neurokraniums erfolgen.

Die fibröse Dysplasie macht 2,5\% aller Knochentumoren aus [4, 6, 18]. Die monoostotische Form der fibrösen Dysplasie

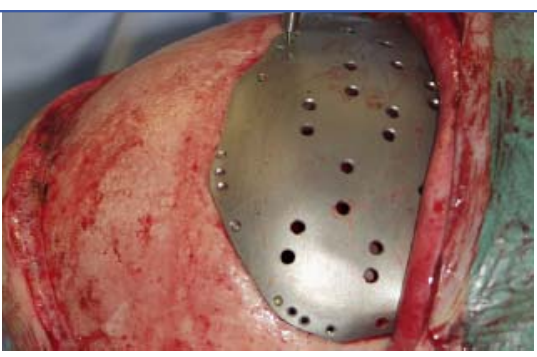

Abb. $6 \triangle$ Einpassen des aus einem postoperativ angefertigten CAS-CT-Datensatz präfabrizierten Titanimplantats (Patientin 2, - Tabelle 1)

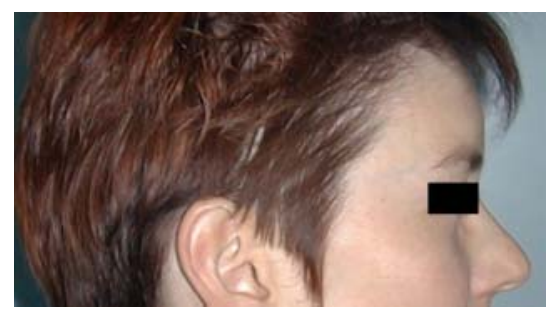

Abb.7 $\triangle$ Patientin eine Woche postoperativ nach Einsetzen des aus einem postoperativ angefertigten CAS-CT-Datensatz präfabrizierten Titanimplantats (Patientin 2, - Tabelle 1)

ist mit 70-80\% die häufigste Verlaufsform $[9,28]$. In 10-30\% der Fälle findet sich eine hemilaterale kraniofaziale Manifestation $[9,16]$. Die Maxilla ist dabei am häufigsten betroffen, gefolgt vom Os frontale, Os sphenoidale und Os zygomaticum. Diagnose und differenzialdiagnostische $\mathrm{Ab}$ grenzung zu anderen Krankheitsbildern erfolgt heutzutage radiologisch anhand eines hochauflösenden Schädel-CT.

Am Schädel lassen sich 3 Formvarianten beschreiben. Die pagetoide Form ist meist am Schädeldach lokalisiert und ist bei der polyostotischen Form der fibrösen Dysplasie häufig, eine sklerosierende Form meist an Schädelbasis und Oberkiefer und eine zystoide Form an Schädeldach und Mandibula. Als Differenzialdiagnosen kommen eine Ostitis deformans Paget in Betracht, wobei der Morbus Paget bei älteren Patienten jenseits des 40. Lebensjahres auftritt, sowie bei vorwiegend sklerosierenden Läsionen ein Meningeom.

Die MR-Bildgebung ist von untergeordneter Bedeutung und wird in der Regel bei unklaren Befunden herangezogen oder im Kindesalter zur Verlaufskontrolle durchgeführt [24]. Vor allem bei raschem Wachstum der Läsion sollte an eine sekundäre Malignisierung in ein osteogenes Sarkom oder Chondrosarkom gedacht wer- 
den [26]. Zur Verlaufsdiagnostik bei Patientin 6 dient das MRT, da aufgrund des Alters der Patientin eine fortwährende Strahlenbelastung der Augenlinse als sensibles Organ im Strahlenfeld vermieden werden soll. In Ausnahmefällen kann die Technetium-99m-Diphosphonat-Knochenszintigraphie über den Aktivitätszustand der fibrösen Dysplasie Auskunft geben und helfen, eine monoostotische Form gegen eine polyostotische Form abzugrenzen [27].

Das histopathologische Bild ist geprägt von einem Ersatz des Knochenmarks durch faserreiches Bindegewebe und exzentrische Atrophie der Kompakta mit fibrozystischer Umbau (Pseudozysten; [6]).

Seit Einführung der Computertomographie mit immer höher auflösenden Verfahren lassen sich heute pathophysiologische Nerven- und Gefäßkompressionen oder Verlegungen nasaler Drainagewege leicht identifizieren und chirurgisch beheben. Lediglich die tumorbedingte Entstellung des äußerlich sichtbaren Splanchnound Neurokraniums oder dessen Deformierung infolge knöcherner chirurgischer Herdsanierungen stellten bislang ein schwieriges Problem da. Da die kraniofaziale fibröse Dysplasie bevorzugt unilateral auftritt, lässt sich unter Nutzung der Körpersymmetrie die korrespondierende Gegenseite als spiegelbildliche Schablone verwenden, um die ursprünglichen Konturen wiederherstellen zu können.

Durch Anwendung der CAS lässt sich im Simulationsmodus eine Schablone herstellen [22] oder der abzutragende überschüssige Knochen intraoperativ einzeln abmessen (• Abb. 1a-d). Ebenso lassen sich Implantate für radikal-chirurgische Defekte mit Hilfe der CAS unter Nutzung präoperativer CT-Datensätze konfigurieren, die dann entsprechend dieser Angaben fabriziert werden und ein- oder zweizeitig schließlich zur Wiederherstellung der ursprünglichen Kontur passgenau eingesetzt werden können (• Abb. 4, 5, 6, 7) $[5,21]$.

Ähnlich könnte die CAS auch bei lokal begrenzten Knochenveränderungen der kraniofazialen fibrösen Dysplasie der Nasennebenhöhlen mit Verlegung der Drainagewege eingesetzt werden, um unter Schonung des umliegenden physiologischen Schleimhautgewebes umschriebe- 
nen begrenzt navigiert kontrolliert die pathologischen Knochenveränderungen zu beseitigen. Dies hätte sich beispielsweise in einem unserer Fälle (Patientin 5, • Tabelle 1) mit einem pathologischen knöchernen Verschluss der Keilbeinhöhle infolge einer knöchern lokal begrenzten kraniofazialen fibrösen Dysplasie angeboten. In diesem Fall ließ sich jedoch bereits bei der präoperativen klinisch-endoskopischen Untersuchung die Vorderwand der Keilbeinhöhle transseptal leicht identifizieren, sodass wir auf eine CAS verzichteten.

Nachteile durch den intraoperativen Einsatz der CAS sind unserer Meinung nach nicht zu erwarten. Vielmehr sehen wir die CAS zur optimalen chirurgischen Behandlung der fibrösen Dysplasie als „hilfreich“ an (gemäß den Kategorien zur Beurteilung der Indikation zur CAS in der NNH-Chirurgie [20]). Auch in Anbetracht der Gesamtoperationszeit bei fibröser Dysplasie ist der zusätzliche Aufwand durch den Einsatz der CAS nach unseren bisherigen Untersuchungen zu vernachlässigen [20]. Die CAS ist in den Händen des erfahrenen Anwenders als zusätzliche Hilfe bei der Durchführung schwierigerer Eingriffe im Bereich der Nasennebenhöhlen und der Schädelbasis anzusehen $[8,14]$.

\section{Fazit für die Praxis}

\section{Die CAS dient als intraoperative Bemes- sungsgrundlage bei der kraniofazialen fi- brösen Dysplasie und stellt ein wertvol- les Instrument zur kontrollierten kosme- tisch-ästhetischen Knochenkorrektur oder zum Konfigurieren geeigneter Im- plantate im Bereich des Splanchno- und Neurokraniums dar.}

\section{Korrespondierender Autor Prof. Dr. J. Schipper}

Universitätsklinik für Hals-, Nasen- und Ohrenheilkunde und Poliklinik, Universitätsklinikum Freiburg, Killianstraße 5, 79106 Freiburg E-Mail: schipper@hno.ukl.uni-freiburg.de

\section{Danksagung}

Unser Dank gilt Herrn Professor Dr. Dr. Egbert Machtens und der Cranio Construct Gesellschaft für individuelle Implantate $\mathrm{mbH}$, Bochum, für die wertvollen Hilfestellungen.

Interessenkonflikt: Der korrespondierende Autor versichert, dass keine Verbindungen mit einer Firma, deren Produkt in dem Artikel genannt ist, oder einer Firma, die ein Konkurrenzprodukt vertreibt, bestehen.

\section{Literatur}

1. Adler CP (1983) Knochenkrankheiten. Thieme, Stuttgart, $S 238$.

2. Albright $F$, Butler $A M$, Hamptom $A O$, Smith $P$ (1937) Syndrome characterized by osteitis fibrosa disseminata, areas of pigmentation and endocrine dysfunction, with precious puberty in females; report of five cases. New Engl J Med 216: 727

3. Beck O, Schramm G, Kyselka R (1972) Leistet die Bestimmung der sauren Mukopolysaccharide und des Hydroxyprolins einen Beitrag zur Differentialdiagnose der fibrösen Dysplasie (Jaffé-Lichtenstein) und der Osteogenesis imperfecta congenita? Beitr Orthop Traumatol 19: 740-742

4. Brugel FJ, Grevers G, VogI TJ (1990) Supraorbitaler Tumor als Manifestation einer fibrösen Dysplasie Jaffé-Lichtenstein. Laryngorhinootologie 69: 533537

5. Bücheler $M$, Weihe $S$, Eufinger $H$, Wehmöller $M$, Bootz F (2002) Rekonstruktion des Os frontale mit individuellen Titanimplantaten nach chirurgischer Therapie der Stirnbeinosteomyelitis. HNO 50: 339346

6. Bühling KJ, Lepenies J, Witt K (Hrsg) (1995) Intensivkurs: Allgemeine und spezielle Pathologie. Urban \& Schwarzenberg, München, S 382

7. Cohen MM, Howell RE (1999) Etiology of fibrous dysplasia and McCune-Albright syndrome. Int J Oral Maxillofac Surg 28: 366-371

8. Ecke U, Khan M, Maurer J, Boor S, Mann WJ (2002) Intraoperative Navigation in der Chirurgie der Nasennebenhöhlen und der vorderen Schädelbasis. Fehlerquellen und Störfaktoren. HNO 50: 928-934

9. Enderle A, Willert HG, Zichner L (1984) Angeborene und erworbene Skelettsystemerkrankungen. In: Witt AN et al. (Hrsg) Orthopädie in Praxis und Klinik, Bd III: Allgemeine Orthopädie, 2. Aufl. Thieme, Stuttgart, S 91-124

10. Fechner RE, Mills SE (Hrsg) (1992) Tumor of the bones and Joints. Atlas of tumor pathology. Am Reg Pathol 1992: 147-151

11. Herzog M, Hoppe F (2000) Der interessante Fall Nr. 36. Fibröse Dysplasie des Felsenbeines (Osteodystrophia fibrosa Jaffé-Lichtenstein). Laryngorhinootologie 79: 447-449

12. Jaffé $H L$, Lichtenstein L (1942) Fibrous dysplasia of bone. A condition affecting one, several or many bones, the gracases of which may present with abnormal pigmentation of skin, premature sexual development, hyperthyroidism or still other extraskeletal abnormalities. Arch Pathol 33: 777-816

13. Jaffé HL, Lichtenstein L (1942) Non-osteogenic fibroma of bone. Am J Pathol 18: 205

14. Khan M, Ecke U, Mann WJ (2003) Der Einsatz eines optischen Navigationssystem in der Nasennebenhöhlenchirurgie. HNO 51: 209-215
15. Lichtenstein L (1938) Polyostotic fibrous dysplasia. Arch Surg 36: 874-898

16. Maassen MM, Plinkert PK, Pockler C (1993) Fibröse Dysplasie Jaffé-Lichtenstein als Ursache von progredienter Protrusio bulbi und temporärem Visusverlust. HNO 41: 491-496

17. Neumann K, Gotze G, Holzhausen HJ (2003) Fibröse Dysplasie des Felsenbeins. HNO 51: 998-1001

18. Prein J, Remagen W, Spiessl B, Uehlinger E (Hrsg) (1985) Atlas der Tumoren des Gesichtsschädels. Springer, Berlin Heidelberg New York, S 97-127

19. Schäfer EL, Sturm A (1963) Zur Therapie und zum Verlauf der fibrösen Knochendysplasie. Dtsch Med Wochenschr 88: 464-467

20. Schipper J, Ridder GJ, Aschendorff A, Klenzner T, Arapakis I, Maier W (2004) Verbessert die computergestützte Navigation in der endonasalen Nasennebenhöhlenchirurgie die Prozess- und die Ergebnisqualität? Laryngorhinootologie 83: 298-307

21. Schipper J, Ridder GJ, Spetzger U, Teszler CB, Fradis M, Maier W (2004) Individual prefabricated titanium implants and titanium mesh in skull base reconstructive surgery. A report of cases. Eur Arch Otorhinolaryngol 261: 282-290

22. Schramm A, Gellrich NC, Gutwald R et al. (2000) Indications for computer-assisted treatment of cranio-maxillofacial tumors. Comput Aided Surg 5 : 343-352

23. Schwindinger WF, Francomano $C A$, Levine $M A$ (1992) Identification of a mutation in the gene encoding the alpha subunit of the stimulatory $G$ protein of adenyl cyclase in McCune Albright syndrome. Proc Natl Acad Sci USA 89: 5152-5156

24. Stiglbauer R, Ritschl P, Kramer J, Imhof H (1989) Fibröse Dysplasie: Erscheinungsbild im Magnetresonanztomogamm. Rofo Fortschr Geb Rontgenstr Neuen Bildgeb Verfahr 151:338-341

25. Stöver T, Schönermark MP, Issing PR (1998) Der interessante Fall Nr. 17. Fibröse Dysplasie Jaffé-Lichtenstein (Osteodystrophia fibrosa). Laryngorhinootologie 77: 596-598

26. Taconis WK (1988) Osteosarcoma in fibrous dysplasia. Skeletal Radiol 17: 163-170

27. Vogelsang H, Stoppler L, Thiede G (1978) Fibröse Dysplasie des Schädel - eine röntgenologische, computertomographische und szintigraphische Studie. Rofo Fortschr Geb Rontgenstr Nuklearmed 128: 253-257

28. Wehner MS, Issing WJ (2000) Der interessante Fall $\mathrm{Nr}$. 37. Fibröse Dysplasie Jaffé-Lichtenstein (Osteodystrophia fibrosa). Laryngorhinootologie 79: 498500

29. Wellmitz G (1977) Zur Ätiopathogenese und Genetik der fibrösen Dysplasie (Jaffe-Lichtenstein). Beitr Orthop Traumatol 24: 84-89

30. Wittwer B (1973) Genetische Fragen des Albrightund des Jaffé-Lichtenstein-Syndroms. Beitr Orthop Traumatol 20: 169-172 